\title{
Collinearly improved impact-parameter dependent Balitsky-Kovchegov evolution
}

\section{Jan Cepila}

Faculty of Nuclear Sciences and Physical Engineering, Czech Technical University in Prague, Czech Republic

\section{Jesus Guillermo Contreras}

Faculty of Nuclear Sciences and Physical Engineering, Czech Technical University in Prague, Czech Republic

\section{Marek Matas*}

Faculty of Nuclear Sciences and Physical Engineering, Czech Technical University in Prague, Czech Republic

E-mail: matas.marek1@gmail.com

\begin{abstract}
The Balitsky-Kovchegov equation has been successfully used to describe a wide range of processes in diffraction, saturation physics and many others. It has a wide use both in phenomenology and theory; its solution (the scattering amplitude) has received significant attention in the past years. However, solutions including the impact-parameter dependence have been shown to exhibit so-called Coulomb tails, which spoil their use in phenomenology, due to an unphysical growth of the target. In this study we show, that choosing the recently proposed collinearly improved kernel suppresses the effect of the Coulomb tails and enables us to describe simultaneously structure function data as well as vector meson photoproduction.
\end{abstract}

XXVII International Workshop on Deep-Inelastic Scattering and Related Subjects - DIS2019 8-12 April, 2019

Torino, Italy

${ }^{*}$ Speaker. 


\section{Introduction}

The high-energy (equivalently small-x) limit of QCD has received significant attention in the past years because of the applicability of the perturbative expansions of this theory, which are allowed by the asymptotic behavior of the strong interaction coupling as well as because of the experimental facilities that were able to provide data to confront predictions given by the theory.

This limit can be described in the color-dipole model with the use of the Balistky-Kovchegov (BK) equation $[1,2]$. This equation has been solved with great success under the approximation that the impact parameter dependence factorizes from the energy evolution. Previous attempts to include the impact parameter dependence to the evolution have been spoiled by the presence of the so-called Coulomb tails, which produce an unphysically large target and violate the MartinFroissart bound $[3,4]$. We show, that using the collinearly improved kernel in the BK equation, the Coulomb tails are heavily suppressed, without the necessity to modify the kernel with cut-off terms, nor to add additional phenomenological soft contributions. The new solutions allow for the correct description of data as well as to predict processes that could be measured at future facilities such as the EIC $[5,6]$.

\section{The Balitsky-Kovchegov equation}

Throughout this work, we assume independence of the scattering amplitude both on the angular orientation w.r.t. the target proton (we assume rotational symmetry of the target) as well as on the angle between the impact parameter vector $\vec{b}$ and the dipole size vector $\vec{r}$. This is a good approximation if one uses an initial condition that does not depend on these angles which is the usual approach for the $b$-dependent BK equation [7]. In this case, the BK equation reads

$$
\begin{aligned}
& \frac{\partial N(r, b ; Y)}{\partial Y}=\int d \vec{r}_{1} K\left(r, r_{1}, r_{2}\right)\left(N\left(r_{1}, b_{1} ; Y\right)\right. \\
& \left.+N\left(r_{2}, b_{2} ; Y\right)-N(r, b ; Y)-N\left(r_{1}, b_{1}, Y\right) N\left(r_{2}, b_{2} ; Y\right)\right),
\end{aligned}
$$

where $\overrightarrow{r_{2}}=\vec{r}-\vec{r}_{1}$ and $\left|\vec{r}_{i}\right| \equiv r_{i}$. Variables $b_{1}$ and $b_{2}$ denote the magnitudes of the impact parameters of the daughter dipoles. The collinearly improved kernel $[8,9,10]$ is given by

$$
K\left(r, r_{1}, r_{2}\right)=\frac{\bar{\alpha}_{s}}{2 \pi} \frac{r^{2}}{r_{1}^{2} r_{2}^{2}}\left[\frac{r^{2}}{\min \left(r_{1}^{2}, r_{2}^{2}\right)}\right]^{ \pm \bar{\alpha}_{s} A_{1}} \frac{J_{1}\left(2 \sqrt{\bar{\alpha}_{s} \rho^{2}}\right)}{\sqrt{\bar{\alpha}_{s} \rho}} .
$$

The value of $A_{1}$ was set to $11 / 12$ and the sign in the third factor is positive when $r^{2}<$ $\min \left(r_{1}^{2}, r_{2}^{2}\right)$ and negative otherwise. $\rho \equiv \sqrt{L_{r_{1} r} L_{r_{2}} r}, J_{1}$ is the Bessel function and $L_{r_{i} r} \equiv \ln \left(r_{i}^{2} / r^{2}\right)$. For the running coupling, we use the smallest dipole prescription: $\alpha_{s}=\alpha_{s}\left(r_{\min }\right)$, where $r_{\min }=$ $\min \left(r_{1}, r_{2}, r\right)$ as in [9].

\section{Initial condition to the impact parameter dependent BK equation}

In order to solve the BK equation one has to provide an initial condition. In our case, we chose a combination of the GBW model [11] for the dipole size dependence, and similar to [12], 


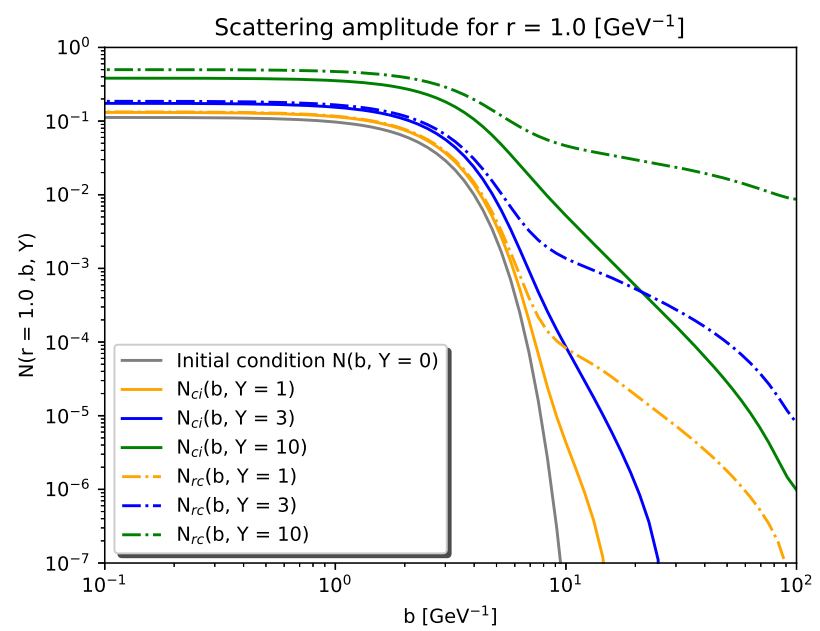

Figure 1: Scattering amplitude with respect to the impact parameter at various rapidities for a dipole of size $r=1 \mathrm{GeV}^{-1}$. Solutions obtained with the running coupling kernel are represented with dashed-dotted lines $\left(N_{r c}\right)$, while solid lines represent solutions with the collinearly improved kernel $\left(N_{c i}\right)$. Figure taken from [15].

a Gaussian distribution for the impact parameter dependence. The considered initial condition can be written as

$$
N(r, b, Y=0)=1-\exp \left(-\frac{1}{2} \frac{Q_{s}^{2}}{4} r^{2} T\left(b_{q_{1}}, b_{q_{2}}\right)\right),
$$

where $b_{q_{i}}$ are the impact parameters of the individual quark and antiquark of the initial bare dipole and

$$
T\left(b_{q_{1}}, b_{q_{2}}\right)=\left[\exp \left(-\frac{b_{q_{1}}^{2}}{2 B}\right)+\exp \left(-\frac{b_{q_{2}}^{2}}{2 B}\right)\right] .
$$

The two parameters $Q_{s}^{2}$ and $B$ in the initial condition have a physical interpretation. The saturation scale $Q_{s}^{2}$ determines the scale at which nonlinear effects become important and $B$ represents the variance of the Gaussian distribution of the target in the impact parameter space.

The BK equation does not have an analytic solution and has to be solved numerically. We have used the Runge-Kutta method of order four with the algorithm described in [13, 14], extended to include the $b$-dependence. Parameters in the initial condition $\left(Q_{s}^{2}\right.$ and $\left.B\right)$ and in the running coupling $\left(C^{2}\right)$ were set to values of $Q_{s}^{2}=0.49 \mathrm{GeV}^{2}, B=3.22 \mathrm{GeV}^{-2}$ and $C=9$ [15].

Fig. 1 shows the impact-parameter dependence of the dipole scattering amplitude for a dipole of size $r=1 \mathrm{GeV}^{-1}$ at various rapidities. It shows both solutions obtained with the runningcoupling as well as with the collinearly improved kernel. We have used the same initial condition for the sake of a valid comparison and we can see, that the Coulomb tails in the large- $b$ region are strongly suppressed with the use of the collinearly improved kernel.

\section{Comparison to data}

We have used the solutions obtained by the $b$-dependent BK equation to compute the structure 

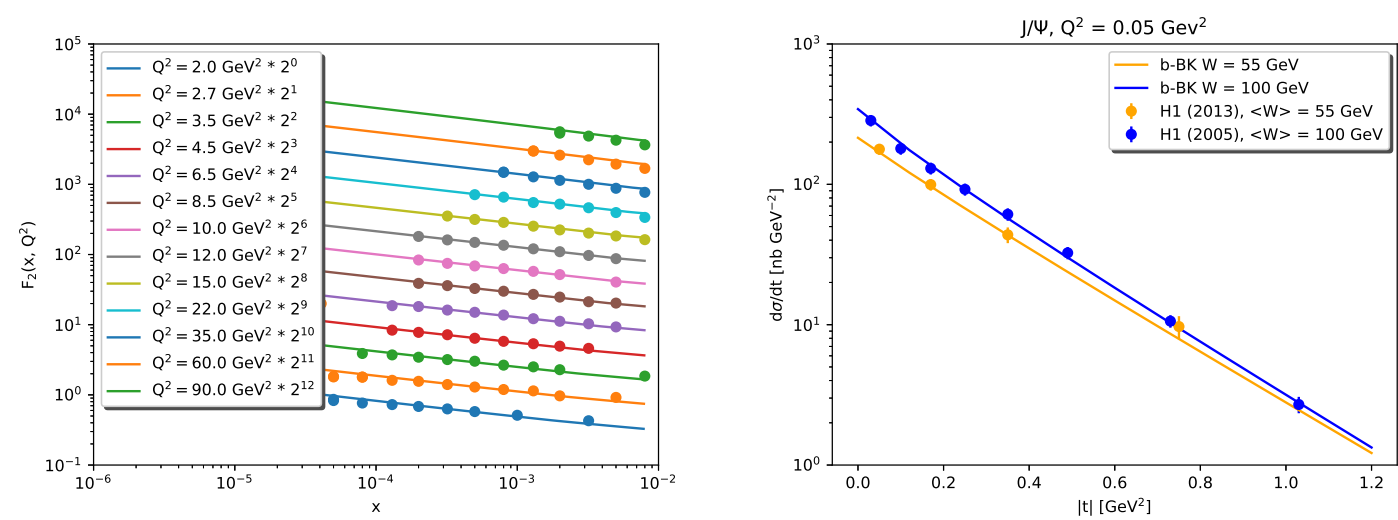

Figure 2: Structure function data from HERA [16] compared to the results of the computation based on solutions to the collinearly improved $b$-dependent BK equation (left). Predicted $|t|$ dependence of the cross section for the exclusive photoproduction of $\mathrm{J} / \psi$ vector mesons off protons compared to data from the $\mathrm{H} 1$ Collaboration at HERA at $\langle W\rangle=55 \mathrm{GeV}$ [17] and $\langle W\rangle=100 \mathrm{GeV}$ [18] (right). Figures taken from [15].

function $F_{2}\left(x, Q^{2}\right)$, which was then compared to HERA data (Figure 2 (left)). The structure function can be expressed in the dipole model with the use of the scattering amplitude as

$$
F_{2}\left(x, Q^{2}\right)=\frac{Q^{2}}{4 \pi^{2} \alpha_{\mathrm{em}}} \sum_{f} \int \mathrm{d} \vec{r} \mathrm{~d} \vec{b} \mathrm{~d} z\left|\Psi_{T, L}^{f}(z, \vec{r})\right|^{2} \frac{\mathrm{d} \sigma^{q \bar{q}}\left(\vec{r}, x_{f}\right)}{\mathrm{d} \vec{b}}
$$

where $\Psi_{T, L}^{f}(z, \vec{r})$ are the wave functions of a photon splitting into a quark-antiquark dipole of flavor $f, \alpha_{\mathrm{em}}$ is the electromagnetic coupling constant and $z$ is the dipole energy fraction carried by the quark. The cross section is related to the scattering amplitude as

$$
\frac{\mathrm{d} \sigma^{q \bar{q}}(\vec{r}, x)}{\mathrm{d} \vec{b}}=2 N(\vec{r}, \vec{b}, x) .
$$

We use $x_{f}=x\left(1+\left(4 m_{f}^{2}\right) / Q^{2}\right)$ with $m_{f}$ set to $100 \mathrm{MeV} / c^{2}$ for light quarks. The mass of the charm quark was fixed to $1.3 \mathrm{GeV} / c^{2}$ as in [9].

We have also computed the $|t|$ dependence of cross section for the exclusive photoproduction of $\mathbf{J} / \psi$ vector meson. The computation was carried out at fixed values of $W$ (Figure 2 (right)). The prescription for the amplitude of this process is given by (see e.g. [19])

$$
A\left(x, Q^{2}, \vec{\Delta}\right)_{T, L}=i \int \mathrm{d} \vec{r} \int_{0}^{1} \frac{\mathrm{d} z}{4 \pi}\left(\Psi^{*} \Psi_{\mathrm{J} / \psi}\right)_{T, L} \int \mathrm{d} \vec{b} e^{-i(\vec{b}-(1-z) \vec{r}) \cdot \vec{\Delta}} \frac{\mathrm{d} \sigma^{q \bar{q}}}{\mathrm{~d} \vec{b}}
$$

where $T$ and $L$ represent transverse and longitudinal photons, $-t \equiv \vec{\Delta}^{2}$ and $\Psi_{\mathrm{J} / \psi}$ is the wave function of the dipole fluctuating into a $\mathrm{J} / \psi$ vector meson. We have used the boosted Gaussian wave functions [20,21] with parameters as determined in [19].

The $|t|$-differential cross section is then obtained by squaring the amplitude and dividing it by $16 \pi$, where transverse and longitudinal contributions are added. The cross section is corrected for the contribution of the real part of the dipole scattering amplitude and for the fact that the two 
gluons have different momentum, which is known as the skewedness correction [22] (for more details see discussion in Sec. 3 of [19]).

\section{Summary}

In this work we have solved the impact-parameter dependent Balitsky-Kovchegov equation with the collinearly improved kernel. We have demonstrated, that unlike in the case with the running coupling kernel, the so-called Coulomb tails (unphysical growth of the scattering amplitude in the large $b$ region) are heavily suppressed, which enables the correct description of both the structure function data $F_{2}\left(x, Q^{2}\right)$ as well as the data for cross section for the $|t|$ dependence of exclusive photoproduction of $\mathrm{J} / \psi$ vector mesons off protons. The data are described without the need of inserting additional parameters to the computation such as additional soft contributions or massive infrared regulators to the kernel. The scattering amplitude obtained this way can be then used in phenomenology to predict various observables that are within the reach of future planned electron-ion colliding facilities.

Acknowledgments. We would like to thank Dagmar Bendová, Heikki Mäntysaari and Cyrille Marquet for fruitful discussions. Our work has been partially supported by grant 17-04505S of the Czech Science Foundation, GAČR and the COST Action CA15213 THOR. Computational resources were provided by the CESNET LM2015042 grant and the CERIT Scientific Cloud LM2015085, provided under the program "Projects of Large Research, Development, and Innovations Infrastructures".

\section{References}

[1] I. Balitsky. Operator expansion for high-energy scattering. Nucl. Phys., B463:99-160, 1996.

[2] Jamal Jalilian-Marian, Alex Kovner, Andrei Leonidov, and Heribert Weigert. The Wilson renormalization group for low x physics: Towards the high density regime. Phys. Rev., D59:014014, 1998.

[3] Krzysztof J. Golec-Biernat and A. M. Stasto. On solutions of the Balitsky-Kovchegov equation with impact parameter. Nucl. Phys., B668:345-363, 2003.

[4] Jeffrey Berger and Anna Stasto. Numerical solution of the nonlinear evolution equation at small $\mathrm{x}$ with impact parameter and beyond the LL approximation. Phys. Rev., D83:034015, 2011.

[5] A. Accardi et al. Electron Ion Collider: The Next QCD Frontier. Eur. Phys. J., A52(9):268, 2016.

[6] J.L. Abelleira Fernandez et al. A Large Hadron Electron Collider at CERN: Report on the Physics and Design Concepts for Machine and Detector. J.Phys., G39:075001, 2012.

[7] Jeffrey Berger and Anna M. Stasto. Exclusive vector meson production and small-x evolution. JHEP, 1301:001, 2013.

[8] E. Iancu, J. D. Madrigal, A. H. Mueller, G. Soyez, and D. N. Triantafyllopoulos. Resumming double logarithms in the QCD evolution of color dipoles. Phys. Lett., B744:293-302, 2015.

[9] E. Iancu, J. D. Madrigal, A. H. Mueller, G. Soyez, and D. N. Triantafyllopoulos. Collinearly-improved BK evolution meets the HERA data. Phys. Lett., B750:643-652, 2015. 
[10] Leszek Motyka and Anna M. Stasto. Exact kinematics in the small x evolution of the color dipole and gluon cascade. Phys. Rev., D79:085016, 2009.

[11] Krzysztof J. Golec-Biernat and M. Wusthoff. Saturation effects in deep inelastic scattering at low Q**2 and its implications on diffraction. Phys. Rev., D59:014017, 1998.

[12] Larry D. McLerran and Raju Venugopalan. Boost covariant gluon distributions in large nuclei. Phys. Lett., B424:15-24, 1998.

[13] Jan Cepila and Jesus Guillermo Contreras. Rapidity dependence of saturation in inclusive HERA data with the rcBK equation. arXiv:1501.06687, 2015.

[14] Marek Matas, Jan Cepila, and Jesus Guillermo Contreras Nuno. Numerical precision of the solution to the running-coupling Balitsky-Kovchegov equation. EPJ Web Conf., 112:02008, 2016.

[15] J. Cepila, J. G. Contreras, and M. Matas. Collinearly improved kernel suppresses Coulomb tails in the impact-parameter dependent Balitsky-Kovchegov evolution. Phys. Rev., D99(5):051502, 2019.

[16] F. D. Aaron et al. Combined Measurement and QCD Analysis of the Inclusive e+- p Scattering Cross Sections at HERA. JHEP, 01:109, 2010.

[17] C. Alexa et al. Elastic and Proton-Dissociative Photoproduction of J/psi Mesons at HERA. Eur. Phys. J., C73(6):2466, 2013.

[18] A. Aktas et al. Elastic J/psi production at HERA. Eur. Phys. J., C46:585-603, 2006.

[19] H. Kowalski, L. Motyka, and G. Watt. Exclusive diffractive processes at HERA within the dipole picture. Phys. Rev., D74:074016, 2006.

[20] J. Nemchik, Nikolai N. Nikolaev, and B. G. Zakharov. Scanning the BFKL pomeron in elastic production of vector mesons at HERA. Phys. Lett., B341:228-237, 1994.

[21] J. Nemchik, Nikolai N. Nikolaev, E. Predazzi, and B. G. Zakharov. Color dipole phenomenology of diffractive electroproduction of light vector mesons at HERA. Z. Phys., C75:71-87, 1997.

[22] A. G. Shuvaev, Krzysztof J. Golec-Biernat, Alan D. Martin, and M. G. Ryskin. Off diagonal distributions fixed by diagonal partons at small x and xi. Phys. Rev., D60:014015, 1999. 